\title{
Motor conduction block and high titres of anti- GM1 ganglioside antibodies: pathological evidence of a motor neuropathy in a patient with lower motor neuron syndrome
}

\author{
David Adams, Thierry Kuntzer, Andreas J Steck, Alexander Lobrinus, \\ Robert C Janzer, Franco Regli
}

\begin{abstract}
A patient with a progressive lower motor neuron syndrome had neurophysiological evidence of motor axon loss, multifocal proximal motor nerve conduction block, and high titres of anti-ganglioside GM1 antibodies. Neuropathological findings included a predominantly proximal motor radiculoneuropathy with multifocal IgG and IgM deposits on nerve fibres associated with a loss of spinal motor neurons. These findings support an autoimmune origin of this lower motor neuron syndrome with retrograde degeneration of spinal motor neurons and severe neurogenic muscular atrophy.
\end{abstract}

(F Neurol Neurosurg Psychiatry 1993;56:982-987)

Interest in the pathological role of anti-ganglioside antibodies has been stimulated by the identification of monoclonal $\operatorname{IgM}$ antibodies reacting with specific gangliosides in patients with motor neuron diseases and monoclonal gammopathy. ${ }^{1-4}$ Polyclonal anti-GM1 antibodies have also been found in the absence of a gammopathy in patients with motor neuron syndromes, particularly in a subgroup of patients with multiple motor neuropathy and lower motor neuron syndrome. ${ }^{5-9}$ The presence of these antibodies raises the possibility of an ongoing autoimmune process in patients with motor neuron disease. In a few of these patients improvement in strength has occurred after reduction of antibody titres by treatment with immunosuppressive drugs. 5910 Anti-GM1 antibodies may bind to the nodes of Ranvier, ${ }^{11}$ but the significance of this antibody binding pattern and its role in nerve conduction block remain to be determined. ${ }^{9}$

Centre Hospitalier Universitaire Vaudois, Lausanne, Switzerland

Service de Neurologie D Adams T Kuntzer A J Steck F Regli

Institut Universitaire de Pathologie A Lobrinus R C Janzer

Correspondence to: Professor A Steck, Service de Neurologie, CHUV, 1011 Lausanne, Switzerland.

Received 12 February 1992 and in final revised form 8 December 1992

Accepted 11 December 1992 and temporal dispersion included a more than $20 \%$ reduction in the negative CMAP amplitude and an increase in the negative duration of no more than $15 \%$ of the maximum CMAP elicited by stimulation at the proximal site compared with that evoked by distal stimulation. ${ }^{12}$ In cases where stimulation could not be applied proximally, a conduction block was admitted when the number of motor unit potentials evoked by graded stimulation was much greater than could be obtained during maximal voluntary contraction or when the total area of the CMAP was greater than the sum of the areas of the individual motor unit potentials. ${ }^{13}$ Motor nerve conduction studies were measured over long segments at conventional sites (Erb's point, axilla, elbow, wrist) and also over short segments for the ulnar nerve. $F$ wave latencies from at least 10 responses were recorded following stimulation at the wrist. Using surface electrodes, sensory nerve action potentials were recorded orthodromically. Needle examination was performed using standard Dantec concentric electrodes.

\section{ENZYME LINKED IMMUNOSORBENT ASSAY}

The presence of anti-ganglioside GM1 antibodies in serum was measured by the enzyme linked immunosorbent assay (ELISA) using a technique previously described. ${ }^{8}$ The activity against other gangliosides GM2, GM3, GD1a, GD1b, asialo-GM1 was also evaluated. The purified gangliosides were a kind gift of Dr A Martelli (Fidia Research Laboratories, Abano Terme, Italy).

PATHOLOGICAL STUDY

Brain, spinal cord and roots, several peripheral nerves, and muscles were fixed for three weeks in a $10 \%$ buffered paraformaldehyde solution. Forty samples from relevant regions were routinely processed. Paraffin embedded and frozen sections were stained with haematoxylin and eosin, van Gieson and Luxol and Nissl and Luxol stains. Additionally, muscles were stained with periodic acid Schiff, reticulin, Congo red, muscle enzyme, and lipid stains. Immunochemical stains were performed with the following commercial available antibodies against phosphorylated neurofilaments, ubiquitin, glial fibrillary acidic protein (GFAP), lysozyme, myelin basic protein (MBP) and myelin associated glycoprotein (MAG), and anti-IgG, IgM, and IgA antibodies. Samples of spinal roots were also postfixed in glutaraldehyde, epon embedded, and $1 \mu \mathrm{m}$ sections stained with toluidine blue. 


\section{Case report}

A 71 year old man first experienced painful cramps in the thighs and calves on exertion at the end of 1983. Past medical history was not relevant and there was no family history of similar illness. Over the ensuing three years, the patient developed progressive paraparesis, at first characterised by asymmetrical (left more than right) difficulties in climbing stairs; then a bilateral foot drop appeared. In 1985, he needed a stick to walk; in summer 1986 he could only walk with the assistance of two canes. Over the next six months, the weakness extended to the hands and he fell frequently. In December 1986 on examination the cranial nerves were normal but there was a moderate atrophy of shoulders and intrinsic hand muscles and diffuse wasting of the lower limbs. Fasciculations were observed in proximal muscles. The muscle strength was weak and asymmetrical, left side more than the right side, MRC Grade $3+$ to $4 / 5$ distally, $4+15$ proximally in the upper limbs, and 2 to $4 / 5$ distally, 2 to $3 / 5$ proximally in the lower limbs. Sensory responses to light touch, pinprick, vibration, and joint position sense were normal. Deep tendon reflexes were absent in the left leg and present but decreased in the other limbs. Plantar responses were neutral on the right side and questionable on the left. A general examination revealed oedema of the legs up to the knees. There was physiological evidence of diffuse denervation reinnervation by needle EMG. The distal motor and sensory nerve conduction velocities and amplitudes of lower and upper limb sensory nerve action potentials were normal. A diagnosis of probable motor neuron disease was made.

The weakness progressed in the arms and he was found to be tetraplegic in October 1988. In November 1989 he was found to have high anti-ganglioside GM1 antibodies and was reinvestigated. Examination revealed a severe hypotonic tetraplegia: in his arms and legs, only slight fingers and toes flexion could be seen. He also had weakness of the abdomi- nal and respiratory muscles, but the cranial nerves remained normal. There was no sensory deficit or symptoms. Deep tendon reflexes were all absent, plantar responses were neutral, and no sphincter dysfunction or fasciculations were seen. On general examination diffuse oedema of the four limbs was noted.

Biochemical studies were within the normal range, including serum electrophoresis and immunoelectrophoresis, triiodothyronine, thyroxine thyroid stimulating hormone, and immunoelectrophoresis. Serological tests for syphilis, anti-DNA antibodies, CSF, and blood Lyme serology were normal or negative.

The patient was treated with chlorambucil, 9 mg daily but no improvement was observed over the next four months. Death occurred six years after onset. The necropsy was performed 48 hours after death.

\section{Results}

\section{NEUROPHYSIOLOGY}

The results of the nerve conduction studies are summarised in the table. There were motor conduction blocks in four nerves out of the eight tested on both sides, all of them located at the right Erb's point. The degree of the block was more than $50 \%$ for the radial and ulnar nerves. For the axillary and musculocutaneous nerves the number of motor unit potentials evoked by graded stimulation was greater than could be obtained during maximal voluntary contraction. The blocks were associated with a low distal amplitude of the CMAP, expressing axonal loss. Despite the low distal amplitude which leads to difficulty in measuring the block, the proximal and distal responses were of the same shape and could be superimposed: the difference in duration of CMAPs did not exceed $10 \%$ between distal and proximal responses. The differences in amplitude were thus not simply due to increased dispersion of CMAPs. The conduction velocity for the remaining nerve fibres was nearly normal or diminished by less

Table Results of conduction studies in motor and sensory fibres

\begin{tabular}{|c|c|c|c|c|c|}
\hline \multirow[b]{2}{*}{ Nerve } & \multicolumn{2}{|c|}{ Amplitude $(\mu V)$} & \multirow{2}{*}{$\begin{array}{l}\text { Distal latency } \\
\text { (ms) }\end{array}$} & \multirow{2}{*}{$\begin{array}{l}\text { Ratio proximal/distal } \\
\text { amplitude (\%) }\end{array}$} & \multirow{2}{*}{$\begin{array}{l}\text { Conduction velocity } \\
\text { (range, } \mathrm{m} / \mathrm{s} \text { ) }\end{array}$} \\
\hline & Proximal & Distal & & & \\
\hline \multicolumn{6}{|l|}{ Motor: } \\
\hline Right ulnar ${ }^{1 \star}$ & 0 & 310 & 5 & 0 & $44-55 \cdot 7$ \\
\hline Right radial ${ }^{2 \star}$ & 20 & 110 & $6 \cdot 4$ & $18 \cdot 1$ & $39-45$ \\
\hline Right musculocutaneous * & 640 & ND & $6 \cdot 2$ & ND & ND \\
\hline Right axillary ${ }^{\star}$ & 300 & ND & $7 \cdot 4$ & ND & ND \\
\hline Right median & ND & 0 & & & \\
\hline Left median ${ }^{3}$ & 150 & 180 & $5 \cdot 6$ & 83 & $35 \cdot 8-46$ \\
\hline Left musculocutaneous & 0 & ND & & & \\
\hline \multirow{5}{*}{$\begin{array}{l}\text { Left ulnar } \\
\text { Sensory (right side): } \\
\text { Median digit I to wrist } \\
\text { Median palm to wrist } \\
\text { Ulnar digit V to wrist } \\
\text { Radial digit I to wrist }\end{array}$} & 160 & 180 & $6 \cdot 4$ & 88 & $38-45$ \\
\hline & $8 \cdot 4$ & & 3 & & 45 \\
\hline & $28 \cdot 5$ & & 1.5 & & 53.3 \\
\hline & 6 & & 3 & & 45 \\
\hline & $7 \cdot 9$ & & 3 & & $41 \cdot 6$ \\
\hline
\end{tabular}

*Conduction block at Erb's point (see discussion).

$\mathrm{ND}=$ Not done or not measured.

$0=$ No response could be recorded despite different stimulators and position of the patient.

'For ulnar nerve, multiple sites of stimulation included wrist, below and above elbow, axilla and Erb's point while recording CMAPs over abductor digiti minimi muscle. Amplitude ratio was calculated between the most proximal and distal site of stimulation. Between axilla and wrist, no significant drop in amplitude was seen. The nerve conduction velocity within the segment above-below elbow was normal.

${ }^{2}$ For the radial nerve, both needle and disc electrodes were used in an attempt to record the same shape of evoked response. Stimulation sites included forearm, external bicipital fossa, axilla, and Erb's point while recording the CMAPs over extensor indicis proprius muscle. Between axilla and forearm, no significant changes in amplitude was seen.

${ }^{3}$ For median nerve, stimulation sites included wrist and elbow while recording CMAPs over abductor pollicis brevis muscle. 
Figure 1 Spinal cord at C5 level showing cellular details of lower motor neurons. (A) Central chromatolysis (Nissl and Luxol, bar $=50 \mu \mathrm{m}$ ). (B) Diffuse expression of phosphorylated neurofilament proteins (immunoperoxidase method for phosphorylated neurofilament, bar $=100$ $\mu \mathrm{m})$.

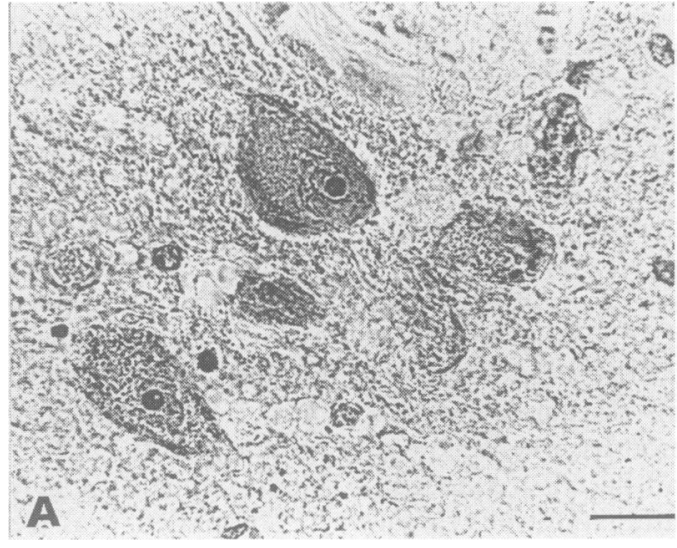

than $30 \%$ of the normal value and no evidence of superimposed focal neuropathy was found (see legend of table). No $F$ waves could be recorded. The sensory nerve action potentials were normal in both upper and lower limbs. By needle EMG, fibrillations and positive sharp waves were recorded at rest in proximal and distal muscles. Insertional activity was not increased, and myokymic or complex repetitive discharges were not seen. In proximal muscles, asynchronous and arrhythmic long duration fasciculation potentials were recorded. During weak effort there were prominent signs of reinnervation with prolonged, enlarged, and polyphasic motor unit potentials in widely distributed muscles. The number of motor unit potentials was reduced during maximum effort.

ELISA

Anti-ganglioside GM1 antibodies were found in high titre in the serum (788 units, normal $<20)$. Anti-ganglioside GD1a and anti-ganglioside GM3 antibodies were also found in the serum but in a lower titre (below 100 units). The anti-ganglioside GM1 antibodies were of IgG class.

\section{PATHOLOGICAL STUDY}

The general necropsy findings included a

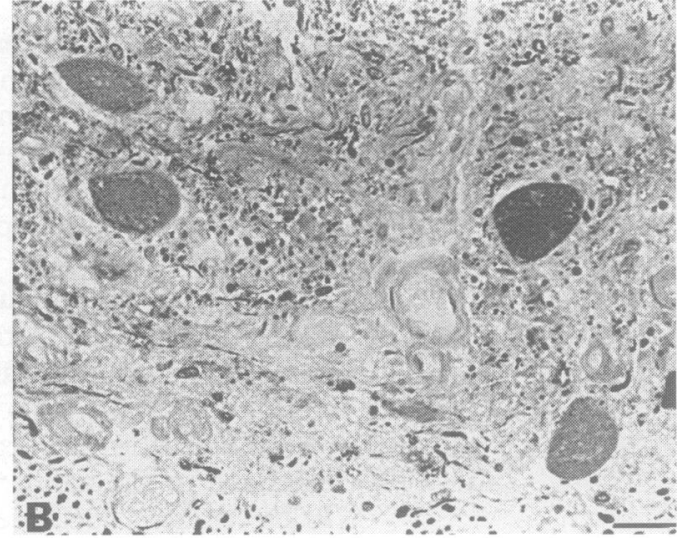

bilateral diffuse pulmonary emphysema with consecutive predominantly right sided cardiac hypertrophy and a terminal acute bronchopneumonia.

Macroscopically the significant neuropathological lesions included atrophic anterior roots and a generalised muscular atrophy. Brain (1330 g), spinal cord, and peripheral nerves appeared normal.

Microscopically the most important lesions were confined to the anterior horn motor neurons (fig 1A, B), anterior roots (fig 2), motor peripheral nerves (figs 3 and 4) and muscles, showing severe neurogenic atrophy. The severity of the lesions in the spinal cord and the anterior roots was more marked at the lumbosacral and lower thoracic levels and showed less involvement of the upper thoracic and cervical segments, suggesting a slowly progressive and ascending course of the disease. The lesion of the spinal cord consisted of a loss of up to $60 \%$ of the motor neurons in the most severely affected lower segments, accompanied by a reactive gliosis, while in the upper segments the presence of central chromatolytic figures and the pathological accumulation of phosphorylated neurofilaments in the perikaryon of motor neurons (fig 1), devoid of ubiquitin inclusions, suggested a retrograde lesion of the motor neurons. Other portions of the spinal
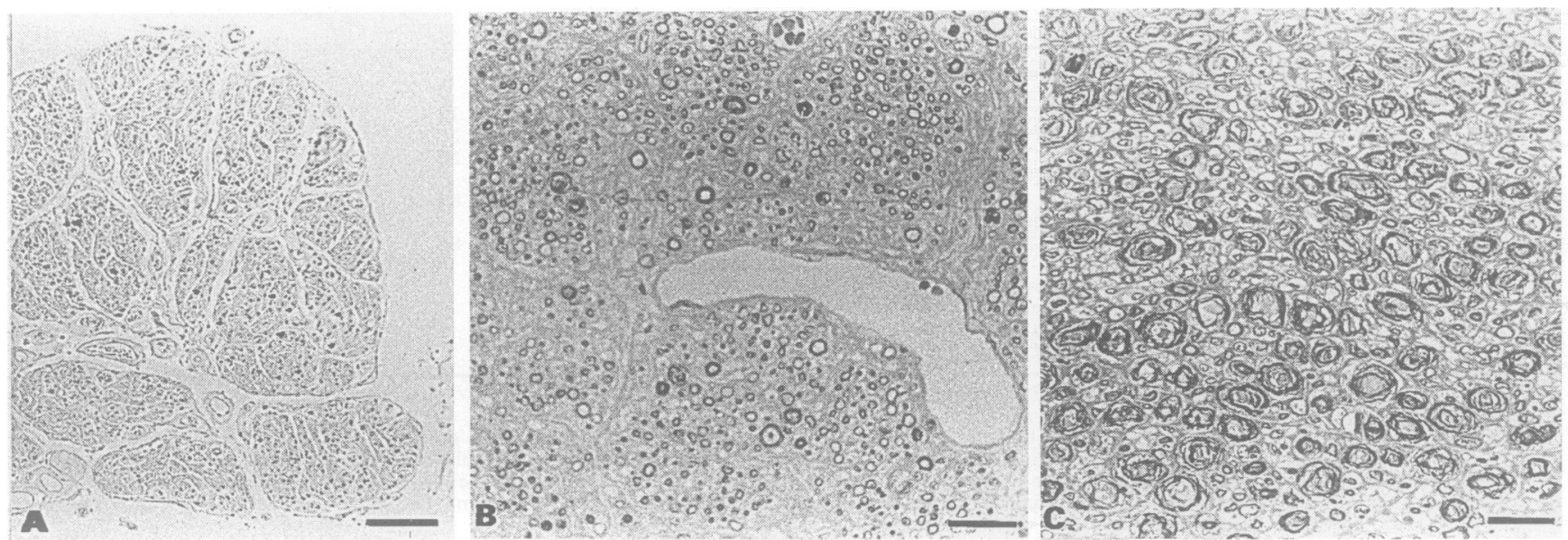

Figure 2 Anterior root pathology at lower thoracic level. (A) Severe nerve fibre loss (immunoperoxidase method for neurofilament protein, bar = 100 um). $(B)$ and $(C) 1 \mu \mathrm{m}$ epon embedded sections stained with toluidine blue (bar $=25 \mu \mathrm{m}$ ), showing severe loss of myelinated nerve fibres in anterior root and relative preserved myelinated nerve fibres in posterior root at the same level $(C)$. Distension of myelin sheaths is caused by postmortem artefact. 


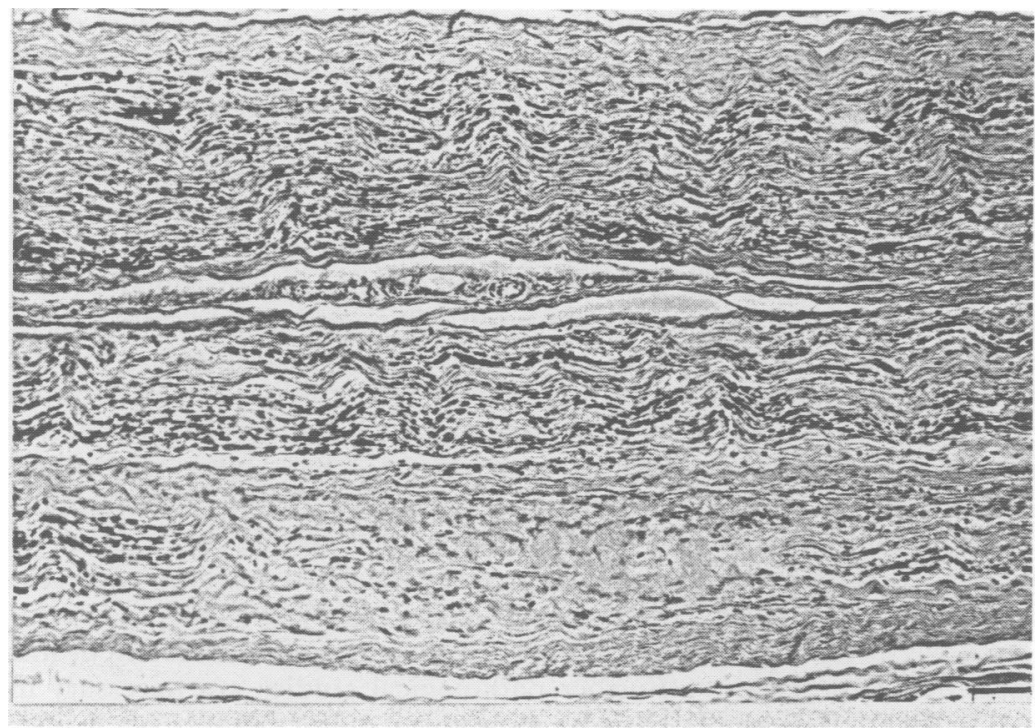

Figure 3 Pathology of peripheral nerve. Fascicular nerve fibres loss in the sciatic nerve (immunoperoxidase method for neurofilament protein, bar $=100 \mu \mathrm{m}$ ).

grey matter were intact as were the ascending and descending tracts, including the corticospinal tract. The retrograde nature of the observed lesion was supported by the finding of an important loss of anterior root fibres (fig $2 \mathrm{~A}, \mathrm{~B})$ accompanied by a severe gliosis in the intraspinal portion and a proliferation of Schwann cells and fibrosis in the extraspinal portion. Myelin loss, as shown by myelin basic protein (MBP) and myelin associated glycoprotein (MAG) immunohistochemistry, was not more pronounced than nerve fibre loss. Axonal ballooning was not observed. The immunohistochemical results for IgG and IgM deposits in the spinal cord were inconclusive.

All the structures of the brainstem and the brain were intact; all cranial motor nerve nuclei and the primary motor cortex espec-

Figure 4 (A) Proximal sciatic nerve fibres with multifocal IgM deposits (IgG deposits not shown) in endoneurial compartment (arrows) and around vessels (arrowheads). (B) Serial step section from same sample as illustrated in (A) showing no multifocal staining with a control incubation.
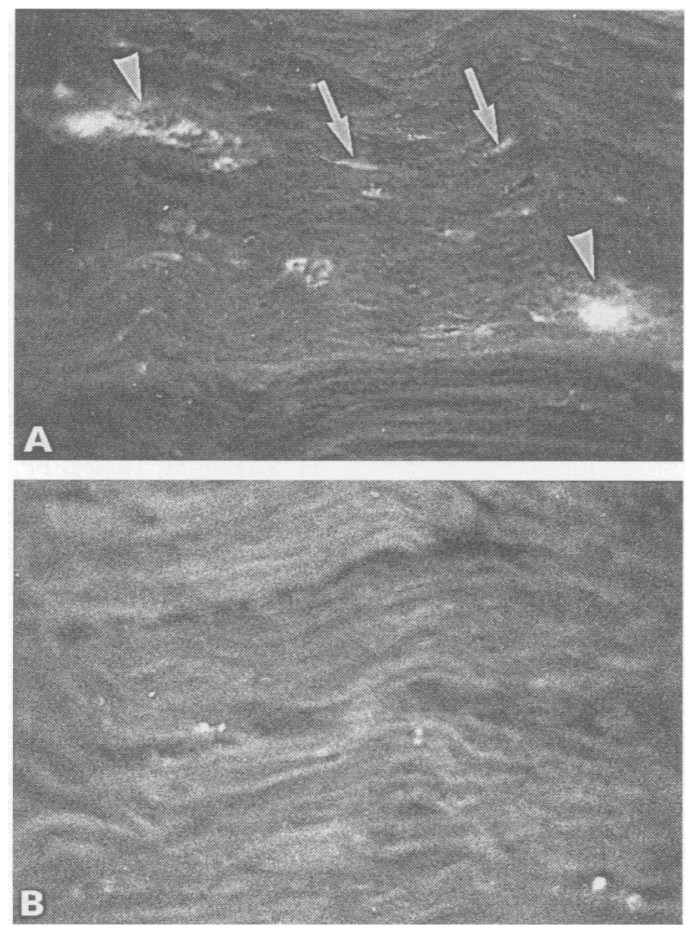

ially showed no neuronal loss, neuronal inclusions, or gliosis. The corticospinal tract showed no axonal loss or demyelination or any other significant lesion at all the levels examined, including corona radiata, internal capsule, mesencephalic peduncle, pontine and bulbar portions, and along the spinal cord. Pure motor peripheral nerves were not examined, but the large mixed sensorimotor sciatic and peroneal nerves showed severe pathological changes clearly affecting some fascicles more than others (fig 3 ). There was severe axon depletion with loss of myelin and a reactive proliferation of Schwann cells and fibrosis. Immunohistochemistry performed on unfixed frozen samples of the same nerves showed multifocal deposits of IgM (fig 4) and IgG, but not IgA, in relatively well preserved fascicles on the nerve fibres.

\section{Discussion}

The slow progression, lack of brisk reflexes, and absence of bulbar dysfunction were clinical features that helped to distinguish our case from amyotrophic lateral sclerosis. Antibodies to ganglioside GM1 are not usually found in patients with presumably nonimmune lower motor neuron syndromes such as spinal muscular atrophy. ${ }^{7-9}$ High titres of serum anti-ganglioside GM1 antibodies ( $>4$ $S D$ above the mean value), are found in some motor neuron disease and peripheral motor neuropathy, whereas low titres $(<100$ in our laboratory) are less specific and may be found in sera from patients with a variety of neurological and autoimmune disorders. ${ }^{89}$ Two motor syndromes may be considered in our patient: a multifocal motor neuropathy and a lower motor neuron syndrome. ${ }^{9}$ These syndromes may overlap with similar clinical and immunological features, but in lower motor neuron syndrome no motor conduction block may be seen on physiological studies, ${ }^{9}$ and these patients may develop widespread weakness and atrophy suggestive of a spinal muscular atrophy and a progressive course leading to death.

Recent papers have underscored that a drop in the recorded CMAP amplitude from proximal to distal stimulation is insufficient to recognise conduction block and alternative interpretations should be considered. ${ }^{13}$ In our patient, we did not find abnormal desynchronisation that could result in a reduction of the CMAP. Insufficient stimulus intensity is unlikely because we stimulated Erb's point with different stimulators and differing the position of the patient without modifying the amplitude of the evoked responses. The interpretation of these findings is difficult when the usual physiological consequences of demyelination, such as slight conduction delay and prolonged $F$ responses, are absent. ${ }^{12} \mathrm{We}$ postulate that the proximal conduction blocks in our patient could be due to the severity of the axonal loss with an increased excitation threshold in the remaining motor nerve fibres. The onset of the disease in the legs and the severity of the axonal 
loss, with "axonal" proximal conduction blocks on physiological studies make the diagnosis of lower motor neuron syndrome more likely than multifocal motor neuropathy.

Conditions involving a primary motor neuropathy with secondary degeneration of motor neurons or a primary motor neuron disorder with secondary axonal degeneration have to be considered in the light of the pathological study. We found a predominantly motor, proximally accentuated, radiculoneuropathy with multifocal IgG and IgM deposits on the nerve fibres and a slowly ascending retrograde degeneration of the spinal motor neurons with a corresponding severe, partly endstage neurogenic muscular atrophy.

A primary spinal motor neuron disease seems to be unlikely in our case, since the severity of the observed changes were more accentuated in the anterior roots, followed by the peripheral nerves and finally by the changes in the anterior horn cells. In favour of a primary axonal lesion are the presence of evenly distributed phosphorylated neurofilaments within the perikarya of spinal motor neurons with central chromatolysis, suggesting impaired axonal transport, ${ }^{14}$ and deposits of immunoglobulins on the nerve fibres. We cannot provide definitive evidence that the immunoglobulin deposits are at or near the nodes of Ranvier, since myelin stained adjacent sections or myelin stains performed on sections previously stained by immunofluorescence, or even double immunofluorescence staining, were not conclusive. What can reasonably be said is that there is multifocal deposition of $\operatorname{IgG}$ and $\operatorname{IgM}$ on nerve fibres and that this pattern is compatible with the localisation at or near the nodes of Ranvier as described in experimental models. We did not find prominent demyelinative features, onion bulbs, or inflammatory cells in peripheral nerves. The conclusions regarding the specificity of cytoplasmic inclusions and Ig deposits in our case must be regarded with caution in view of the small number of necropsies that have been reported in spinal muscular atrophy and pure motor neuropathies,,$^{14} 15$ and further investigations of the role of anti-GM1 antibodies in peripheral nerve are needed to elucidate the exact structure to which they react.

High anti-gangliosides GM1 titres have been shown to be relatively specific for patients with lower motor neuron disease, ${ }^{136916}$ motor neuropathies, ${ }^{5678916}$ or, more rarely, sensorimotor neuropathy. ${ }^{716}$ Gangliosides are important components of nerve membrane in the peripheral and central nervous system, concentrated at the nodes of Ranvier ${ }^{17}$ and at synaptic terminals. ${ }^{18}$ It is interesting to note that IgM deposits have been localised to the nodes of Ranvier in a patient presenting with anti-GM1 antibodies and multifocal motor conduction block. ${ }^{11}$ It is, however, not clear under which conditions the antibodies gain access to their targets and why there is a selective vulnerability of motor nerves. ${ }^{9}$ The antigen might be more concentrated or more accessible in motor nerves than in sensory nerves: a recent study of gangliosides GM1 in peripheral nerves suggested that there were different lengths of hydrophobic moieties of gangliosides GM1 between motor and sensory axons. ${ }^{19}$ Further evidence of interaction with the motor system was provided by the demonstration that anti-gangliosides GM1 antibodies bind to nerve terminals. ${ }^{20}$ In our case, deposits of IgM could be demonstrated in peripheral nerves, but not on the surface of motor neurons, as has been previously demonstrated. ${ }^{11}$ These findings additionally favour the probable autoimmune origin of this lower motor neuron syndrome with secondary neurogenic muscle atrophy and retrograde degeneration of spinal motor neurons. Further pathological studies are necessary in patients with lower motor neuron syndromes with high titre of anti-gangliosides GM1 antibodies to confirm our findings and to further identify the targets and role of anti-GM1 antibodies.

Presented in part at the third meeting of the European Neurological Society, Lausanne, Switzerland, June 1992.

We are grateful for the excellent technical assistance of Drs JP Baumann and B Bosshard, Institut Neuchâtelois d'Anatomie Pathologique, and of $\mathrm{Dr} J$ Miklossy, Institut Universitaire de Pathologie, CHUV, Lausanne.

1 Freddo L, Yu RK, Latov N, et al. Gangliosides GM1 and GD1b are antigens for IgM M-Proteins in a patient with motor neuron disease. Neurology 1986;36:454-8.

2 Latov N, Hays AP, Donofrio PD, et al. Monoclonal IgM with unique specificity to gangliosides GM1 and GD1b and to lactotetraose associated with human motor neuron disease. Neurology 1988;38:763-8.

3 Nardelli E, Steck AJ, Barkas T, et al. Motor neuron syndrome and Monoclonal IgM with antibody activity against gangliosides GM1 and GD1b. Ann Neurol 1988;23:524-8

4 Nobile Orazio E, Legname G, Daverio R, et al. Motor neuron disease in a patient with monoclonal IgM k directed against GM1, GD1b, and high-molecularweight neural specific glycoproteins. Ann Neurol 1990; 28:190-8.

5 Pestronk A, Cornblath DR, Ilyas AA, et al. A treatable multifocal motor neuropathy with antibodies to GM1 multifocal motor neuropathy with an

6 Pestronk A, Chaudhry V, Feldman EL, et al. Lower motor neuron syndromes defined by patterns of weakness, nerve conduction abnormalities, and high titres of antiglycolipid antibodies. Ann Neurol 1990;27:316-26.

7 Sadiq SA, Thomas FP, Kilidireas K, et al. The spectrum of neurologic disease associated with anti-GM1 antibodies. Neurology 1990;40:1067-72.

8 Adams D, Kuntzer T, Burger D, et al. Predictive value of anti-GM1 ganglioside antibodies in neuromuscular diseases: a study of 180 sera $\mathcal{F}$ Neuroimmunol 1991;32. 223-30.

9 Pestronk A. Invited review: Motor neuropathies, motor neuron disorders, and antiglycolipid antibodies. Muscle Nerve 1991;14:927-36.

10 Shy ME, Heiman-Patterson T, Parry GJ, et al. Lower motor neuron disease in a patient with autoantibodies against $\mathrm{Gal}(1-3) \mathrm{GalNAc}$ in gangliosides $\mathrm{GM} 1$ and GD1b: improvement following immunotherapy Neurology 1990;40:842-4.

11 Santoro M, Thomas FP, Fink ME, et al. IgM deposits at nodes of Ranvier in a patient with amyotrophic lateral sclerosis, anti-GM1 antibodies, and multifocal motor conduction block. Ann Neurol 1990;28:373-7.

12 Feasby TE, Brown WF, Gilbert JJ, Hahn AF. The pathoogical basis of conduction block in human neuropathies. $\mathcal{F}$ Neurol Neurosurg Psychiatry 1985;48:239-44.

13 Cornblath DR, Summer AJ, Daube J, et al. Conduction block in clinical practice. Muscle Nerve 1991;14:869-71.

14 Sobue G, Hashizume Y, Yasuda T, et al. Phosphorylated high molecular weight neurofilament protein in lower motor neurons in amyotrophic lateral sclerosis and other neurodegenerative diseases involving ventral horn cells. Acta Neuropathol 1990;79:402-8.

15 Hays A. Separation of motor neuron diseases from pure motor neuropathies. In: Rowland LP, ed. Amyotrophic motor neuropathies. In: Rowland LP, ed. Amyotrophic Neurology, 1991;56:385-98.

16 Lamb NL, Patten BM. Clinical correlations of antiGM1 antibodies in amyotrophic lateral sclerosis and 
neuropathies. Muscle Nerve 1991;14:1021-7.

17 Ganser AL, Kirschner DA, Willinger M. Ganglioside localization on myelinated nerve fibers by cholera toxin localization on myelinated nerve fibers
binding. $¥$ Neurocytol 1983;12:921-38.

18 Hansson HA, Holmgren J, Svennerholm L. Ultrastructural localization of cell membrane GM1 ganglioside by choleratoxin. Proc Natl Acad Sci USA 1977; 74:3782-6.
19 Ogawa-Goto $\mathrm{K}$, Funamoto $\mathrm{N}$, Abe $\mathrm{T}$, Nagashima $\mathrm{K}$ Different ceramide composition of gangliosides between human motor and sensory nerves. $f$ Neurochem human motor

20 Schluep M, Steck AJ. Immunostaining of motor nerve terminals by IgM $M$ protein with activity against gangliosides GMl and GDlb from a patient with motor neuron disease. Neurology 1988;38:1890-2. 